\title{
Erratum to: On the gluing formula for the analytic torsion
}

\author{
Jochen Brüning • Xiaonan Ma
}

Published online: 19 August 2014

(C) Springer-Verlag Berlin Heidelberg 2014

\section{Erratum to: Math. Z. (2013) 273:1085-1117 DOI 10.1007/s00209-012-1045-5}

In this note, we want to correct a mistake in the proof of [1, Theorem 2.2] which does not affect the statement of the theorem, though. In [1, (2.7)], we incorrectly state an isomorphism of complexes, thus we need to modify the corresponding argument, by first replacing the last six lines on p. 1098, beginning with "we also have ...", with the following text.

"We also have a $\mathbb{Z}_{2}$-equivariant short exact sequence of complexes

$$
0 \rightarrow C^{\bullet}\left(W^{u} / W_{Y_{1}}^{u}, F\right) \otimes \mathbb{C}^{-} \stackrel{\gamma}{\longrightarrow} C^{\bullet}\left(\widetilde{W}^{u}, \widetilde{F}\right) \stackrel{\gamma}{\longrightarrow} C^{\bullet}\left(W^{u}, F\right) \otimes \mathbb{C}^{+} \rightarrow 0,
$$

given by

$$
\gamma\left(\mathfrak{b}^{*} \otimes 1_{\mathbb{C}^{-}}\right)=\frac{\sqrt{2}}{2}\left(\left(\left.j_{1}^{-1}\right|_{X_{1}}\right)^{*} \mathfrak{b}^{*}-\left(j_{2}^{-1} \mid X_{2}\right)^{*} \mathfrak{b}^{*}\right), \quad \gamma\left(\mathfrak{a}^{*}\right)=\frac{\sqrt{2}}{2}\left(\left.j_{1}^{*} \mathfrak{a}^{*}\right|_{X_{1}}+\left.j_{2}^{*} \mathfrak{a}^{*}\right|_{X_{2}}\right) \otimes 1_{\mathbb{C}^{+}},
$$

with $X_{i}=j_{i}(X)$, which induces a $\mathbb{Z}_{2}$-equivariant short exact sequence

$$
0 \rightarrow H^{\bullet}\left(X, Y_{1}, F\right) \otimes \mathbb{C}^{-} \stackrel{\gamma}{\longrightarrow} H^{\bullet}(\tilde{X}, \widetilde{F}) \stackrel{\gamma}{\longrightarrow} H^{\bullet}(X, F) \otimes \mathbb{C}^{+} \rightarrow 0 . ”
$$

Then replace $[1,(2.11)]$ by

$$
\gamma\left(\mathfrak{a}^{*}\right)=\sqrt{2} \mathfrak{a}^{*} \otimes 1_{\mathbb{C}^{+}}
$$

The online version of the original article can be found under doi:10.1007/s00209-012-1045-5.

J. Brüning $(\varangle)$

Institut für Mathematik, Humboldt-Universität zu Berlin, Rudower Chaussee 25, 12489 Berlin, Germany e-mail: bruening@mathematik.hu-berlin.de

X. Ma

UFR de Mathématiques, Université Paris Diderot-Paris 7, Case 7012,

75205 Paris Cedex 13, France

e-mail:ma@math.jussieu.fr 
and $[1,(2.15)]$ by

$$
\begin{aligned}
\log \left(\|\mu\|_{\operatorname{det}\left(H^{\bullet}(\tilde{X}, \widetilde{F}), \mathbb{Z}_{2}\right)}^{M, \nabla f}\right)(g)= & -\frac{1}{2} \log (2) \sum_{x \in B \cap Y_{1}}(-1)^{\operatorname{ind}(x)} \operatorname{rk}(F) \\
& +\log \left\|\gamma_{1}^{-1} \mu_{1}\right\|_{\operatorname{det} H^{\bullet}(X, F)}^{M, \nabla f}+\chi(g) \log \left\|\gamma_{2}^{-1} \mu_{2}\right\|_{\operatorname{det} H^{\bullet}\left(X, Y_{1}, F\right)}^{M, \nabla f}
\end{aligned}
$$

Finally, replace the bottom of p. 1100 from [1, (2.20)] on by:

"for $\sigma_{1} \in H^{\bullet}(\widetilde{X}, \widetilde{F})^{+}, \sigma_{2} \in H^{\bullet}\left(X, Y_{1}, F\right)$,

$$
\begin{aligned}
& \left.P_{\infty} \circ \widetilde{\phi}_{1} \circ P_{\infty}^{-1}\left(\sigma_{1}\right)\right|_{C_{\bullet}\left(W^{u}, F^{*}\right)}=\left.\frac{\sqrt{2}}{2}\left(\sigma_{1}+\phi^{*} \sigma_{1}\right)\right|_{C_{\bullet}\left(W^{u}, F^{*}\right)}=\left.\gamma \sigma\right|_{C_{\bullet}\left(W^{u}, F^{*}\right)}, \\
& \left.\left(P_{\infty} \circ \widetilde{\phi}_{2} \circ P_{\infty}^{-1} \circ \gamma\right)\left(\sigma_{2} \otimes 1_{\mathbb{C}^{-}}\right)\right|_{C_{\bullet}\left(W^{u} / W_{Y_{1}}^{u}, \widetilde{F}^{*}\right)}=\left.\sigma_{2}\right|_{C_{\bullet}\left(W^{u} / W_{Y_{1}}^{u}, \widetilde{F}^{*}\right)} .
\end{aligned}
$$

Set

$$
\tau_{ \pm}=\gamma_{ \pm} \circ P_{\infty} \circ \widetilde{\phi} \circ P_{\infty}^{-1}: H^{\bullet}(\widetilde{X}, \widetilde{F})^{ \pm} \rightarrow H^{\bullet}(\widetilde{X}, \widetilde{F})^{ \pm},
$$

with $\gamma_{+}=\gamma_{1}, \gamma_{-}=\gamma_{2}$. By (2.20), we get

$$
\tau_{ \pm}=\mathrm{Id} . "
$$

\section{Reference}

1. Brüning, J., Ma, X.: On the gluing formula for the analytic torsion. Math. Z. 273(3-4), 1085-1117 (2013) 